\title{
Sustainable Pest Management for Health and Well-Being
}

\author{
Qiyong $\operatorname{Liu}^{1, *}$
}

Pests can not only cause nuisances and allergic reactions and transmit a range of diseases, but they can also pose serious threat to other aspects of life such as the environment, food, agriculture, crops, etc. Over time, the pest control industry has made great progress but has encountered some new challenges and gaps.

To adapt to global changes effectively, pest management strategies are changing constantly. The concept has transitioned from integrated pest management (IPM) to sustainable pest management (SPM) to provide the optimal solution. China, as the world's largest developing country, has continuously contributed innovative ideas to the pest management industry to promote sustainable development of pest management globally. This paper focuses on pests and their adverse impacts, especially in the health field, the challenges and gaps in pest management, the conceptual transition in pest management, and future perspectives.

\section{Pests and Their Adverse Impacts}

Pests are organisms that damage human life, production, and even survival. They can cause significant losses in lives and property and also great loss in other fields such as agriculture, food, environment, and ultimately have a negative impact on people's health and well-being. Pests can be roughly divided into seven categories based on their adverse impacts (Table 1).

On the basis of the World Health Organization's (WHO) report, $80 \%$ of the world's population is at risk of one or more vector-borne diseases (VBDs), and $17 \%$ of the global burden of communicable diseases and over 700,000 deaths are due to VBDs. Malaria causes an estimated 219 million cases globally and results in more than 400,000 deaths every year. More than 3.9 billion people in over 129 countries are at risk of contracting dengue with an estimated 40,000 deaths every year. More over, there are other VBDs including mosquito-borne diseases such as Chikungunya fever, Zika virus infection, yellow fever, West Nile fever, and Japanese encephalitis as well as tick-borne encephalitis. Of note, some VBDs such as Chagas disease (transmitted by triatomine bugs), leishmaniasis (sandflies) and schistosomiasis (snails) affect hundreds of millions of people worldwide.

In China, vectors transmit 10 notifiable VBDs in 3 categories [category A: plague; category B: hemorrhagic fever with renal syndrome (HFRS), epidemic encephalitis B, dengue, leptospirosis, schistosomiasis, and malaria; and category $\mathrm{C}$ : epidemic and endemic typhus, visceral leishmaniasis (kala-azar), and filariasis], 10 non-notifiable and common VBDs (tick-borne forest encephalitis), Xinjiang hemorrhagic fever, Lyme disease, bartonellosis, Zika virus infection, human ehrlichiosis, anaplasmosis, Tsutsugamushi disease, Chikungunya, severe fever with thrombocytopenia syndrome (SFTS), and many other kinds of NTDs or emerging VBDs.

For the adverse impacts of pests in other fields, the Food and Agriculture Organization of the United Nations (FAO) reports that up to $40 \%$ of food crops are destroyed by plant diseases and pests every year. This has resulted in food shortages for millions of people and has caused negative impacts on the main sources of income for poor rural communities by resulting in production and trade losses. In recent years, China's grain production has been reduced by about 40 million tons annually due to pests, which can supply the annual grain consumption of about 80 million people or about $5.3 \%$ of China's total population.

Pest can also cause serious losses in animal husbandry. Through biting, Tabanus horseflies can directly reduce the body weight of cattle by $0.1-1 \mathrm{~kg}$ per day and lead to the reductions in production resulting in huge economic losses. Ticks can spread babesiosis, and theileriosis (East Coast feeder) among livestock causing significant economic losses. In 1999, the animal husbandry losses caused by ticks in Tanzania reached 55 million US dollars.

Based on the FAO's report in 2010, nearly 40 million hectares of forest were affected by plant diseases and insect pests every year. In recent years, the annual average occurrence area of forest pests in China is 120 thousand square kilometers, and the annual 


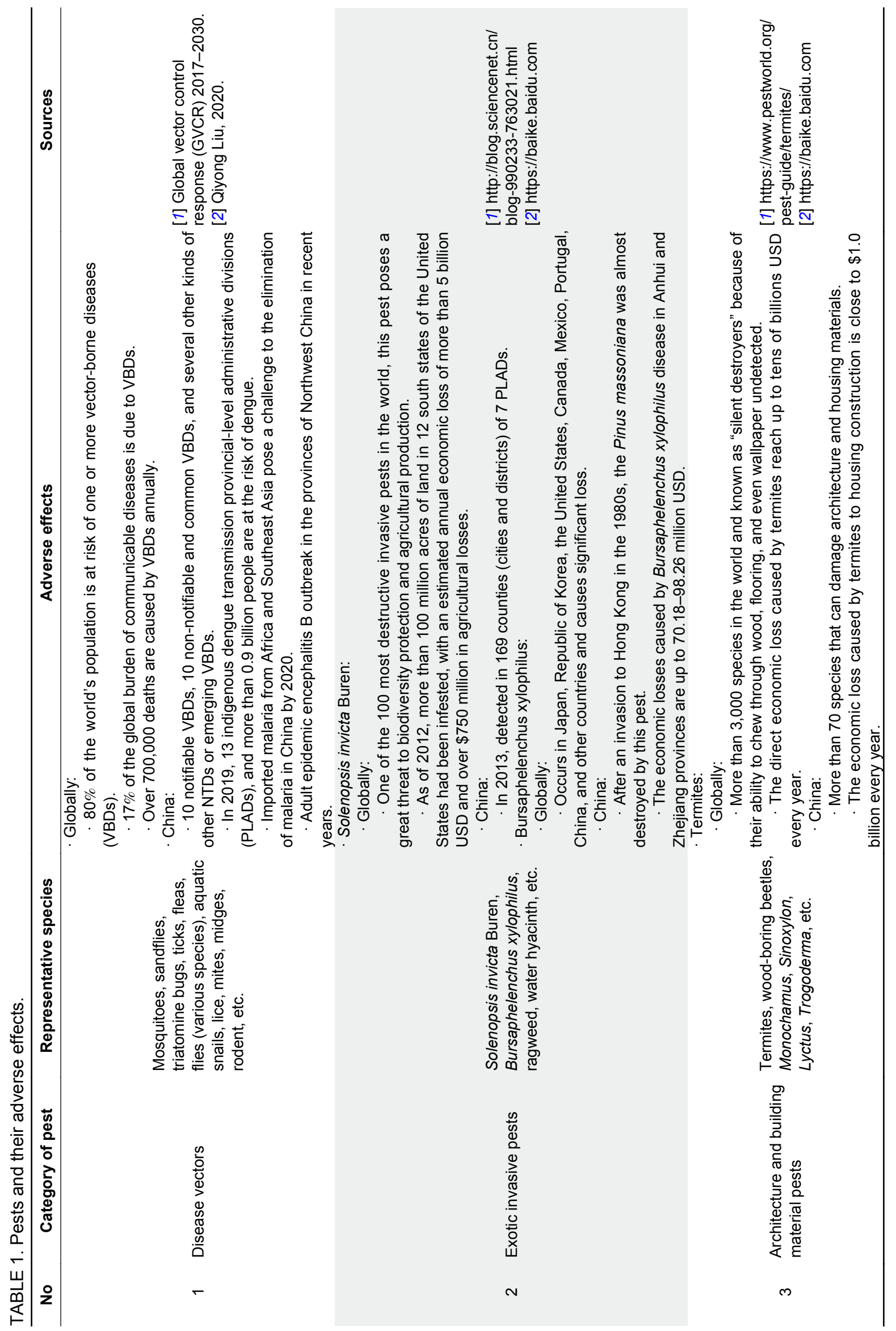




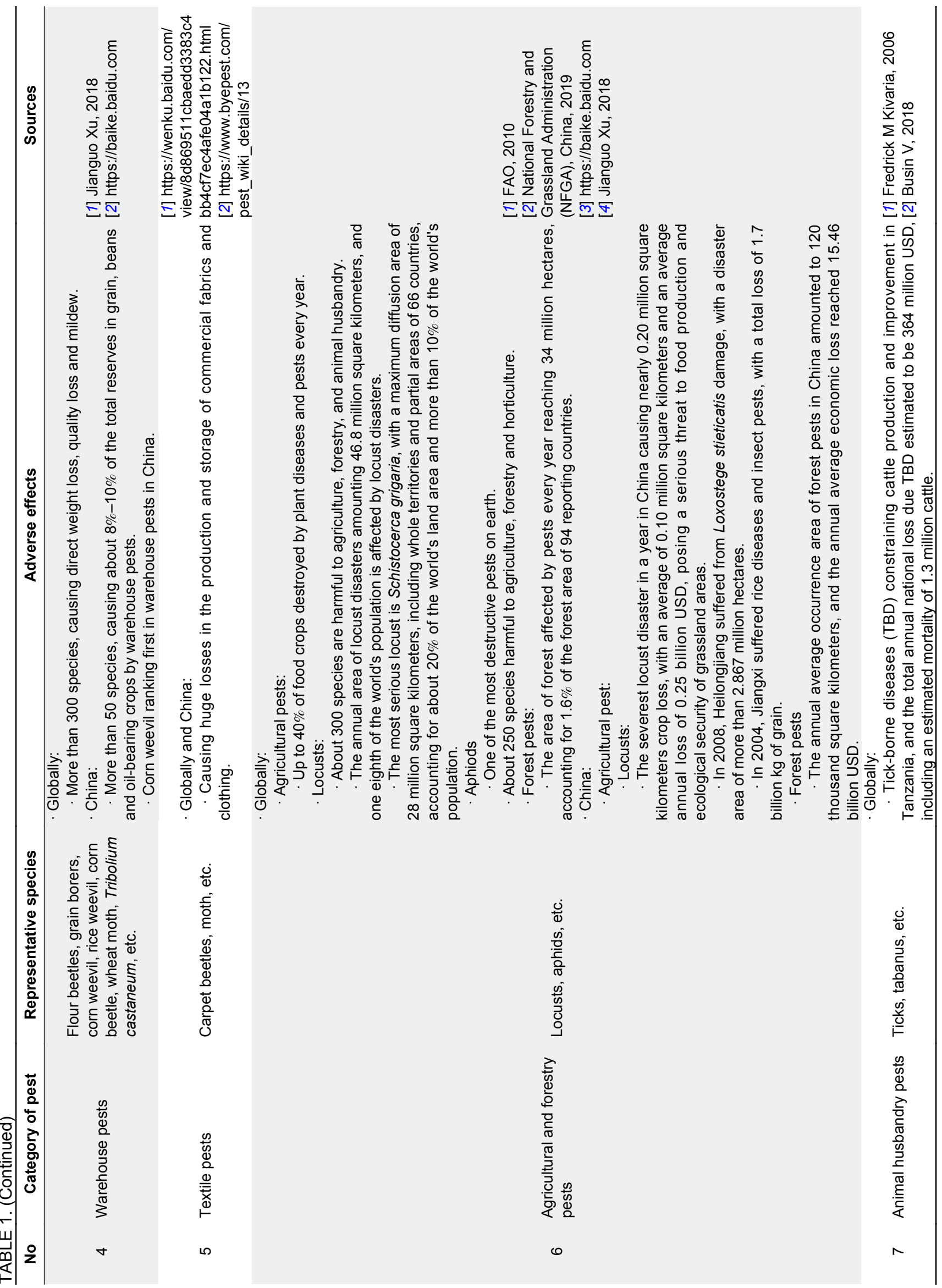


average economic loss is 15.45 billion USD.

\section{Challenges and Gaps Towards Pest Management}

In recent years, climate and environmental change, globalization, urbanization, insecticide resistance, human behavior change, etc., pose new challenges for pest management causing unfavorable effects to the health and well-being of humans.

Changes in vector distribution are being driven by climatic and environmental changes. Globally, the areas and human populations at risk for dengue have recently been increasing significantly due to the spreading of Aedes albopictus and Ae. aegypti resulting from climate change. In China, more than 0.9 billion people currently reside in the risk area of dengue (1), and the risk area and risk populations will increase significantly in the future (2). Due to climate change, mosquitoes in Culex pipiens complex have established its population in Lhasa, Tibet (3). With the changing of the environment, human granulocytic anaplasmosis, a tick-borne disease, was identified in China before 2006 (4). In 2019, the market scale of China's health insecticides reached more than 2.10 billion USD and will continue to grow at a rate of more than $8 \%$. In the future, insecticide resistance will become a more and more serious problem. Outside of China, an unprecedented change in the status of VBDs in Europe has occurred in the early $21^{\text {st }}$ century due to increased globalization (5).

In addition, there are still many deficiencies and gaps concerning capacity building such as insufficient ability for the fast identification of pests, lack of hightech pest surveillance and control tools, lack of selfprotection awareness against pests, etc.

\section{Transition of Pest Control Strategy: from IPM to SPM}

IPM is a science-based, strategic approach for managing insects, rodents, or other vectors. It uses a variety of pest management techniques that focus on pest prevention, pest reduction (G), and the elimination of conditions that lead to pest infestations. Integrated vector management (IVM) is a key component of IPM (7). Though IPM is a comprehensive approach to pest management, its noticeable deficiencies are its lack of planning and systematization towards pest management, more involvement in the technicalities of the method, and being time and energy consuming. Therefore, an innovative pest management concept is urgently needed to guide the pest management practice.

SPM was first proposed by Professor Qiyong Liu in the Pest Management Industry Global Summit, which took place on May 25-27, in Barcelona, Spain in 2010. SPM is designed to meet society's current and future needs for the protection of human health and the environment for the production of food, feed, and fiber and for the use of natural resources. SPM stresses on systematic and sustainable pest management through pest surveillance, risk assessment and alert, control planning, good pest control practice, monitoring and evaluation. It combines a range of pest management practices, including judicious use of pesticides to ensure that our natural resources are utilized efficiently and conserved for future generations. It also addresses the economic viability of available and new pest control products and practices and the planning and systematic nature of pest management. In recent years, VBDs posed great challenges to existing vector control strategies and measures, and mitigation of VBDs will increase economic prosperity by reducing poverty, decreasing productivity losses due to death and disability, and reducing inequality in health and economic outcomes.

Take Aedes control for dengue management in China for example. The process of dengue control under the guidance of SPM (with SVM as the core) is as follows (8): first, carry out Aedes surveillance in a timely and effective fashion; second, conduct a feasibility risk assessment and control planning of Aedes and dengue; and third, choose environmentally friendly techniques and measures comprehensively and in order to implement surveillance-based Aedes control and management consistently. During the process, conduct coordinated activities with multisectoral collaboration and public participation, and suppress the Aedes density below the threshold of dengue transmission (Breteau index or Mos-ovitrap index $<5$ ) (9). This method promoted the transition from reactive outbreak response to proactive outbreak risk reduction of Aedes-borne diseases in China.

As a core component of SPM, SVM was cited by the "Guidelines for dengue prevention and control (2014)" from the China CDC and "Western Pacific Regional Action Plan for Dengue Prevention and Control (2016)" from the WHO. Other WHO guidelines also reference SVM including "The global strategy for dengue prevention and control (2012-2020)" and "Global Vector Control Response 2017-2030" (10), etc. 


\section{SPM for Health and Well-Being}

In the future, we will confront challenges and gaps continuously, and some new challenges may also emerge (11). Pests will become increasingly harmful and will adversely affect public health and well-being. Therefore, current challenges need to be addressed, existing deficiencies must be made up, and SPM should be carried out. Global and regional cooperation, multisectoral joint action and multidisciplinary integration should be strengthened, and the participation of the whole society should be advocated to actualize the sustainable control of pests and the promotion human health and well-being.

In the long run, SPM is likely to be beneficial for the reduction of diseases and poverty, protection of the environment, elimination of hunger, promotion of economic development, and ultimately contributing to human health and well-being and the achievement of sustainable development goals (SDGs).

Funding: This study was supported by the National Natural Science Foundation of China (No. 81703280) and Emergency Response Mechanism Operation Program, National Institute for Communicable Disease Control and Prevention, Chinese Center for Disease Control and Prevention (No. 1310311 02000180007).

doi: $10.46234 / \mathrm{ccdcw} 2020.112$

\# Corresponding author: Qiyong Liu, liuqiyong@icdc.cn.

\footnotetext{
State Key Laboratory of Infectious Disease Prevention and Control, Collaborative Innovation Center for Diagnosis and Treatment of Infectious Diseases, WHO Collaborating Centre for Vector Surveillance and Management, National Institute for Communicable Disease Control and Prevention, Chinese Center for Disease Control and Prevention, Beijing, China.
}

Submitted: May 10, 2020; Accepted: May 20, 2020

\section{REFERENCES}

1. Liu QY. Dengue fever in China: new epidemical trend, challenges and strategies for prevention and control. Chin J Vector Biol Control 2020;31(1):1 - 6. http://dx.doi.org/10.11853/j.issn.1003.8280.2020. 01.001. (In Chinese).

2. Fan JC, Liu QY. Potential impacts of climate change on dengue fever distribution using RCP scenarios in China. Adv Climate Change Res 2019;10(1):1 - 8. http://dx.doi.org/10.1016/j.accre.2019.03.006.

3. Liu QY, Liu XB, Cirendunzhu, Woodward A, Pengcuociren, Bai L, et al. Mosquitoes established in Lhasa city, Tibet, China. Parasites Vectors 2013;6:224. http://dx.doi.org/10.1186/1756-3305-6-224.

4. Zhang LJ, Liu Y, Ni DX, Li Q, Yu YL, Yu XJ, et al. Nosocomial transmission of human granulocytic anaplasmosis in China. JAMA 2008;300(19):2263 - 70. http://dx.doi.org/10.1001/jama.2008.626.

5. Medlock JM, Leach SA. Effect of climate change on vector-borne disease risk in the UK. Lancet Infect Dis 2015;15(6):721-30. http://dx.doi.org/10.1016/S1473-3099(15)70091-5.

6. Zha C, Wang CL, Buckley B, Yang I, Wang DS, Eiden AL, et al. Pest prevalence and evaluation of community-wide integrated pest management for reducing cockroach infestations and indoor insecticide residues. J Econ Entomol 2018;111(2):795 - 802. http://dx.doi.org/ $10.1093 /$ jee/tox356.

7. WHO. Global strategic framework for integrated vector management. Geneva: World Health Organization, 2004

8. Liu QY. Challenge to vector control and sustainable vector management strategy. Chin J Epidemiol 2012;33(1):1 - 8. http://dx.doi.org/10.3760/cma.j.issn.0254-6450.2012.01.001. (In Chinese).

9. Liu QY. Sustainable vector management strategy and practice: achievements in vector-borne diseases control in new China in the past seventy years. Chin J Vector Biol Control 2019;30(4):361-6. http://dx.doi.org/10.11853/j.issn.1003.8280.2019.04.001. (In Chinese).

10. WHO. Global vector control response 2017-2030. Geneva: WHO, 2017.

11. Liu QY, Xu WB, Lu S, Jiang JF, Zhou JP, Shao ZJ, et al. Landscape of emerging and re-emerging infectious diseases in China: impact of ecology, climate, and behavior. Front Med 2018;12(1):3-22. http://dx.doi.org/10.1007/s11684-017-0605-9. 